\title{
Die Kunst des EU-Lobbyings
}

\author{
Rinus van Schendelen
}

Die erste deutschsprachige Übersetzung des Buches „More Machiavelli in Brussels“ von Rinus van Schendelen ist 2012 unter dem Titel „Die Kunst des EU-Lobbyings" erschienen. Dieser Artikel ist eine Zusammenstellung einiger zentraler Passagen aus dem Werk . Es behandelt neue, intelligente Techniken der Einflussnahme, welche zunehmend bisherige Lobbying-Strategien ablösen. Wie man dabei am besten vorgeht, wird speziell bezogen auf Brüssel und die EU gezeigt. Public Affairs Management (PAM) und Europäische Union (EU) sind die Schlüsselbegriffe, die effektive und effiziente Beeinflussung von Institutionen und Behörden ist Kernaufgabe des PAM. Der Autor beschäftigt sich mit der Anwendung des PAM in der EU und erläutert, wie man das Interesse eines Stakeholders in Kommission und Parlament gewinnt und stärkt sowie in jedem Fall verhindert, dass seine Position geschwächt wird.

Bibliografische Angaben: Rinus van Schendelen (2012). Die Kunst des EU-Lobbyings. Erfolgreiches Public Affairs Management im Labyrinth Brüssels. Berlin: Lexxion Verlag. Übersetzt von Susanne Tiedemann. ISBN 978-3-86965-194-1, 478 Seiten, $€ 49,80$.

\section{EU-Lobbying als Public Affairs Management}

Jedes politische System hat seine scheinbaren Komplexitäten, die, will man es beeinflussen, eine Menge Ehrgeiz, Studium und Umsicht erfordern. Dies ist besonders zutreffend für das EU-Spielfeld. Zahlreiche Spieler, die aus verschiedenen Ländern kommen, wollen hier ihre Interessen, aus welchen Gründen auch immer, fördern oder wahren - doch, gespalten wie sie sind, tun sie dies in verschiedene Richtungen. Dieser Wettbewerb kann die Effizienz und Effektivität der Bestrebungen eines jeden, Einfluss auszuüben, beschränken. Die EU mit ihren vielen Institutionen, Verfahren und Charakteren wirft ihre eigenen Komplexitäten auf. Die traditionellen Techniken der Einflussnahme haben längst ihre Grenzen unter Beweis gestellt, wie einige Beispiele verdeutlichen. Zwang, der auf etabliertem EU-Recht basiert und gerichtlich geltend gemacht wird, nimmt häufig viel Zeit in Anspruch und ist mit einer nur kurzfristigen Effektivität verbunden, da die Gegner einen Vergeltungsbumerang verursachen können. Einkapselung erfordert stabile Macht und viele Ressourcen, die knapp sind. Befürwortung führt zu wenig mehr als einem Gegenplädoyer, da jeder Anwalt einen Gegner hat und folglich auch leicht in die Enge getrieben werden kann. Die Argumentation, als Gespräch unter Geschäftsleuten, ist in einigen Situationen nützlich, doch selten ausreichend, da die meisten Wettbewerber unterschiedliche Positionspapiere mit gleichwertig logischen Schlussfolgerungen und glaubwürdigen Referenzen, die ihre wirklichen Interessen verbergen, produzieren. Wie im antiken griechischen Drama können dann alle Charaktere mit ihrer Argumentation Recht haben und, nicht gewillt, Kompromisse einzugehen, im letzten Akt tot sein. Hinsichtlich starker Verbündeter und grimmiger Gegner ist die Argumentation sogar noch nutzloser, da erstere bereits überzeugt und letztere unwillig sind, sich überzeugen zu lassen.

Auf der Suche nach immer effizienteren und effektiveren Techniken der Beeinflussung kam der Begriff des Public Affairs Management (PAM) auf [PARG, 1981; Harris und Fleisher, 2005]. Die public affairs (oder lateinisch res publica) einer Interessengruppe sind die Bedrohungen und Gelegenheiten, die sie in der Außenwelt wahrnimmt, kurz gesagt, ihre externe Agenda, die sie zu ihrer Zufriedenheit behandeln und lösen möchte. Diese Agenda umfasst die Interessen, die des Schutzes oder der Förderung durch Beeinflussung der Außenwelt bedürfen. Die Entscheidung hierzu gehört in den Bereich der Strategie und ist daher ein wesentlicher Teil des allgemeinen Managements. Bislang kann das neue Schlagwort PAM als verbesserte Version der alten Idee des Lobbyings oder als neues Etikett auf einer alten Flasche Wein betrachtet werden. Es enthält in der Tat etwas guten alten Wein, insbesondere das gemeinsame Augenmerk auf „Einfluss ausüben oder etwas wunschgemäß verändern“. In diesem Sinne gibt es keinen Unterschied zwischen einer Gruppe, die Lobbying betreibt, und einer, die PAM anwendet.

Die EU ist ein sehr geeignetes System für die Anwendung des PAM und die gemeinsame Hauptstadt Brüssel ist ein immer wichtigerer Ort des Handelns [Van Schendelen, 2008; Dagger und Kambeck, 2007]. Ob Interessengruppen diese Relevanz nun schätzen oder nicht, ist für die Anerkennung des PAM irrelevant. Die Antagonisten sollten mehr noch als die Protagonisten der EU das PAM-Fachwissen lieben, da gegen den Strom zu schwimmen mehr Ehrgeiz, Studium und Umsicht erfordert, als mit dem Strom zu schwimmen. Gleichwohl fühlen sich in der EU viele Interessengruppen befähigt, vom Wunsch beseelt, genötigt oder eingeladen, sich zu beteiligen und irgendwie Einfluss zu nehmen. Da sie nicht „auf dem Menü stehen “ und die Ergebnisse akzeptieren möchten, möchten sie „am Tisch sitzen“. Aufgrund der Vielfalt Europas werden ihre Interessen unausweichlich mit denen von anderen kollidieren und neue Streitfragen aufwerfen. Ob sie nun wollen oder nicht, treiben die Pressure Groups die Europäisierung durch die Erstellung ihrer Agenden voran. Da sie ihre Interessen auf EU-Ebene vorbringen, betrachten sie die EU-Methode offenbar als effektiver und effizienter als irgendeine traditionelle Integrationsmethode. In der EU zeigen sie zudem ein in hohem Maße variantenreiches Einflussverhalten. Viele beginnen aus dem Menü traditioneller Techniken der Beeinflussung diejenigen auszuwählen, die sie für gewöhnlich im eigenen Land anwenden, darunter manchmal sogar illegale oder ille- 
gitime. Überwiegend verlassen sich auch viele auf die mittelalterliche Lobby und müssen die PAM-Methoden erst noch entdecken, um ihre Chance auf Erfolg verbessern zu können. Ohnehin unterscheidet sich das politische System der EU von jedem innerstaatlichen System und erfordert PA-Ansätze, die daran angepasst sind [Siegele, 2007]. Unter dem Druck vieler Faktoren entwickelt sich eine Art Konvergenz der Praktiken der Beeinflussung. Ein Großteil des Lobbyings wird durch die EU gemaßregelt, beispielsweise durch deren Verfahren und Regeln zur Transparenz. Gruppen von Fassadenkletterern werden sehr schnell durch andere Stakeholder und EU-Funktionäre sanktioniert und oft durch Journalisten ertappt. Eine geschlossene Tür oder ein öffentlicher Skandal ist eine ausgezeichnete Lektion. Die multinationale und multikulturelle EU sollte dennoch weiterhin eine gewisse Vielfalt hinsichtlich der Praktiken der Beeinflussung zulassen.

\section{Wie man „Heimatfront“ und „Feldarbeit“ managt: Potenziale und Grenzen}

Wer betreibt Lobbying, warum, wofür und mit welchem Ergebnis? Eine naive Lobbygruppe beantwortet diese Fragen, bevor sie gestellt werden. Sie sieht ihre eigene Gruppe als autonome Einheit, betrachtet ihre Motivation als selbstverständlich und ihre Ziele als ausreichend klar. Sie unterteilt die Ergebnisse in zwei Kategorien: die Verluste, die anderen angelastet werden, und die Gewinne, die ihrer Leistung zu verdanken sind. Im täglichen Leben wird eine naive Lobbygruppe stets andere beschuldigen und ihre eigenen Hände in Unschuld waschen. Im Gegensatz dazu weiß eine professionelle Gruppe, dass ihre interne Organisation immer ein unvollständiges Puzzle darstellt, ihre Motivation ungewiss ist und ihre Ziele voller Dilemmata sind. Die Ursachen für ihre Verluste und Gewinne schreibt sie ihrem eigenem Verhalten und/oder äußeren Akteuren oder Faktoren zu. Sie beabsichtigt, den kausalen Zusammenhang zwischen ihrer Heimatorganisation und den Ergebnissen ihrer PA zu stärken, indem sie kontinuierlich an ihren inneren Angelegenheiten arbeitet. Hierfür wenden viele PAFunktionäre mehr als die Hälfte ihrer Zeit und Energie auf. In der restlichen Zeit befassen sie sich mit der EU und den Stakeholdern. Sie betrachten ihre eigene Organisation - auch Heimatfront genannt - als die komplizierteste Arena von allen, die ein hohes Maß an Ambition, Studium und Umsicht erfordert.

Die Organisation der eigenen Heimatfront ist eindeutig ein schwieriger Job. Tatsächlich wird hierfür möglicherweise die Hälfte der eigenen Energie, die für das PAM in der EU zur Verfügung steht, verbraucht. Sie erfordert ein hohes Maß an geschickter Erfindungsgabe. Niemand kann blindlings Zusammenhalt, Wissen, Ressourcen, Fähigkeiten, ein Image, eine Strategie, Zielsetzungen, Szenarien, Beurteilungen und anderes mehr produzieren. All diese Zutaten, mit denen Erfolg zusammengebraut werden soll, sollten sorgfältig und damit selektiv vorbereitet werden.

Natürlich ist diese vorbereitende Heimarbeit nicht immer ganz perfekt. Übersteigen die Kosten der Perfektion jene der
Imperfektion, dann kann diese Imperfektion gerechtfertigt sein, da das beste hier der Feind des besseren ist. Alles ist eine Frage der Optimierung. Allein der durch die EU-Fristen verursachte zeitliche Druck führt oft dazu, dass die Alternative zu einer nicht perfekt vorbereiteten Handlung nicht in der Perfektion, sondern im Wegfall dieser Handlung besteht, was viel schlimmer sein kann. Glaubt jedoch eine Lobbygruppe, dass ihre Vorbereitung lediglich besser als die ihres besten Konkurrenten ist, dann sollte sie sich damit nicht zufriedengeben, da sie nicht immer darauf zählen kann, am Ende das „Entscheidungsspiel“ zu gewinnen. Stellt die Lobbygruppe fest, dass einige wichtige Konkurrenten ihre Heimatfront besser organisieren, dann muss sie ihre eigene Vorbereitung verbessern. Diese Logik, die für jeden Wettbewerb Gültigkeit besitzt, ist in der Tat die harte Realität des EU-Spielfelds. Mehr als jemals zuvor wollen viele Interessengruppen auf diesem Spielfeld ein gewünschtes Ergebnis erzielen und investieren zu diesem Zweck in die intelligente Vorbereitung daheim.

Das multinationale Modell ist, um dies klarzustellen, nicht das letzte Wort, was die Optimierung der eigenen Heimatfront betrifft. Es sieht gut entworfen aus: integrativ, professionell und effizient. Zudem hat es sich den Ruf erworben, erfolgreich zu sein. Einige seiner Elemente werden nunmehr weithin nachgeahmt, insbesondere der Aufbau eines Brüsseler Büros. Folglich müssen die Anhänger dieses Modells nun viele Fragen der internen Organisation beantworten. Diese reichen von der Erstellung der gemeinsamen Liste und der Inhalte des Mandats bis hin zur Einbeziehung von Stab- und Linienexperten, mit denen man Vor- und Nachgespräche führen muss. Dem MNO-Modell wird zumeist Schritt für Schritt gefolgt: Es beginnt mit einem Verantwortlichen in Brüssel, und Jahre später folgen wichtige Veränderungen im Innersten der heimischen Organisation. Das MNO-Modell ist ein Teil der Vorbereitung auf das Match und stellt noch nicht das eigentliche Match dar. Die EU-Feldarbeit muss noch folgen.

Paradoxerweise kann man, je strenger man diese verschiedenen Fragen und Aktivitäten analytisch voneinander getrennt hält, diese umso besser für die Produktion eines gewünschten EU-Ergebnisses sicher miteinander verflechten. Die größte Kunst besteht darin, alle Fragen und Aktivitäten systematisch miteinander zu verbinden und somit neue Einsichten für die Feldarbeit und die Heimarbeit in Hinblick auf sich selbst und die Arena zu erwerben. Dies perfekt machen zu können, bleibt in der Praxis stets ein Traum. Doch ohne das Streben nach dieser Perfektion kann niemand jemals in die „erste Liga “ aufsteigen. Je mehr Erfahrung man mit Heimarbeit und Feldarbeit sammelt, desto leichter kann man die Verbindungen zwischen den beiden herstellen und desto mehr Expertise bekommt man. Die Belohnung ist eine bessere Wahl bei der nächsten für die Feldarbeit und die Heimarbeit auszuführenden Aktivität, welche die Lobbygruppe dichter an das gewünschte EU-Ergebnis heranführt. Wie in jedem starken Wettbewerb ist es die Marge, die den Unterschied macht.

Durch diese Verbindung erhält man auf Heimarbeit basierende Feldarbeit und auf Feldarbeit basierende Heimarbeit, 
was perfekt zusammen passt. Die vorbereitende Arbeit ist dann genauso sehr eine Quelle der Inspiration, um das Spiel zu spielen, wie das Spielen des Spiels Inspiration für die vorbereitende Arbeit ist. Durch diesen Prozess schafft man auch eine echte F\&E des PAM in der EU. Dies hat sogar noch mehr Optionen, Werkzeuge, Menüs und Dilemmata und zusätzlich noch mehr handhabbare Faktoren und konstruierbare Vektoren zur Folge. Das Überangebot potenzieller Handlungen wird noch größer und ist theoretisch unbegrenzt. Für den Profi ist dies kein unangenehmes Problem, sondern eine angenehme Gelegenheit, die mehr Wege hin zum EU-Erfolg eröffnet. Was in der Theorie unbegrenzt ist, kann jedoch in der Praxis viele Grenzen haben. Dies wird das Thema des nächsten Kapitels sein. Springen wir zu einer paradoxen Schlussfolgerung: Der Profi, der bereits einen größeren Vorteil aus dem Überangebot potenzieller PA-Handlungen hat als der Amateur, leidet unter den Grenzen des PA in der Praxis weniger als der Amateur.

\section{3 Überlegungen vor dem Handeln}

Die neue Einflusstechnik des Public Affairs Managements und insbesondere ihre professionelle Anwendung ist besser an die Realitäten der EU angepasst als die traditionellen Techniken. Sie basiert im Wesentlichen auf dem Respekt für die vielen (häufig irritierenden) verschiedenen Werte und Stakeholder in jeder Arena, die die Macht haben, Vergeltung zu üben, wobei dieser Respekt sich nicht von dem Respekt unterscheidet, den kämpfende Soldaten, die eine riskante Schlacht überleben wollen, ihren bewaffneten Feinden entgegenbringen. Dieser Respekt erhält durch sorgfältige Vorbereitung auf und umsichtiges Verhalten während der Schlacht Substanz. Die Lobbygruppen, die ihre PA ernst nehmen, wollen ebenso überleben. Schlagen sie den Window-out-Weg ein, sind sie gut vorbereitet und handeln umsichtig. Sie suchen inmitten des Labyrinths nach besseren Wegen und auf dem Trampolin der EUEntscheidungsfindung nach besseren Zeitpunkten. Im Gegensatz zu tatsächlichen Soldaten und zu der Ära Machiavellis können sie nicht darauf hoffen, ihre Feinde für immer zu töten. Wenn sie mit Stakeholdern und Funktionären den Windowin-Weg nehmen, betreiben sie Lobbying für einen Frieden durch das Finden eines Kompromisses und das Aushandeln eines Abkommens.

Dieser Ansatz für jede EU-Arena - euphemistisch Spielfeld genannt - erfordert eine Menge an Reflexion hinsichtlich des Handelns. Es gibt so viele Herausforderungen, Optionen, Menüs und Dilemmata, dass im Vergleich dazu das Schachspiel leicht erscheint. An der Heimatfront muss sich die Lobbygruppe mit der wahren Natur der Herausforderungen und der Präsenz solcher Voraussetzungen wie ausreichender Zusammenhalt und gutes Image befassen. Die Umwandlung einer langen Liste von EU-Tagträumen und -Albträumen in eine gemeinsame Liste mit realisierbaren Zielen erfordert ein intensives Studium des Spielfelds. Für jede spezifische Arena muss man die zur Debatte stehenden Streitfragen, die involvierten Stakeholder, die zeitlichen Dimensionen und die Arenagrenzen identifizieren und über deren bestmögliches
Management nachdenken. Die Feldarbeit steckt voller Dilemmata, wie etwa in Bezug auf unterschiedliche Verhaltensstile, den Gebrauch von Lärm und die zeitliche Abstimmung der Aktivitäten. Die Anzahl der potenziellen Akteure, denen man sich nähern kann, der zu benutzenden Faktoren und der zu erzeugenden Vektoren ist stets zu hoch, um diese alle handhaben zu können, und erfordert daher eine kluge Selektion. Der Lobbyprozess ist voller Grenzen, die ebenfalls Aufmerksamkeit erfordern. Lobbygruppen müssen sich all dieser Faktoren bewusst sein und intelligent entscheiden.

Die intelligente Reflexion über das Handeln verstärkt zwar die Chance auf Erfolg, ist jedoch weder notwendig noch ausreichend dafür. Selbst mit gründlicher Reflexion kann eine Lobbygruppe das Opfer eines durch ein unvorhergesehenes Ereignis verursachten Unglücks werden. Besonders bekannte Beispiele hierfür sind der Sturz der Kommission Santer 199899, das Problem des „Terrorismus“ 2001 oder die Bankenkrise 2008. Da ein Unglück immer auftreten kann, bestehen die besten Antworten darauf in einer Szenarienplanung und Umsicht.

Reflexion ist nicht das Ziel, sondern ein höchst nützliches Mittel, um die Chance auf mehr Erfolg in einer spezifischen Arena zu erhöhen. Dies impliziert nicht, dass auf die Reflexion immer Lobbyaktivitäten folgen müssen. Eine Lobbygruppe kann auch zu dem Schluss kommen, dass es besser sein kann, passiv zu bleiben, wenn sie beispielsweise das Kosten-NutzenVerhältnis jeder weiteren Handlung als unattraktiv erachtet. Zumindest ist sie dann mental aktiv, wenn es um das Bewusstsein für die gegenwärtige Situation und ihre Vorbereitung auf jede kommende Veränderung geht. Entscheidet sie, eine aktive Rolle zu spielen, so muss sie sich gründlich vorbereiten, wobei dies von der effektiven Organisation der Heimatfront bis zur sorgfältigen Analyse der Arena reicht.

Sucht sie nach PA-Taktiken und -Techniken, die in Bezug auf Effizienz (Kosten-Nutzen-Verhältnis) und Effektivität (erwünschte Ergebnisse) bestmöglich abschneiden, muss die Interessengruppe erhebliche Investitionen in das Sammeln solider Informationen über ihre eigene Position wie auch über die EU-Arena tätigen. Um Intelligenz aus diesen Informationen ziehen zu können, muss sie über diese reflektieren. Diese Investition ist eine ständige Aktivität, die vor dem Handeln beginnen und währenddessen und danach andauern sollte. Für diese Investition belohnt sich die Lobbygruppe selbst mit besseren Entscheidungen hinsichtlich ihrer geplanten Handlungen, ihrer Reaktionen während des interaktiven Lobbyings und ihrer Verbesserungen für zukünftige Spiele. Die „besseren Taktiken“ sind niemals dauerhaft festgelegt, sondern hängen von der Bewertung der spezifischen Situation, der Strategie und den mögliche Kettenreaktionen, die sich stets verändern, ab. Nützliche Lektionen kann man auch von verschiedenen Lobbygruppen, die Lobbying für dasselbe Dossier betreiben, Personen, die auf der Empfängerseite arbeiten, beobachtenden Journalisten, Kollegen im PAM und Wissenschaftlern, die das Verhalten von Lobbygruppen erforschen, erhalten. Im PAM 
sind die wahren Profis unersättlich in ihrem Verlangen nach verbessertem Wissen und Lernen.

Sie sind zudem aus zwei Gründen umsichtig. Erstens hat jede Arena bestimmte Risiken. Eine Lobbygruppe kann entweder Teil der gewinnenden oder der verlierenden Koalition sein. Während des Arenaprozesses kann sie alte Freunde verlieren, in eine feindselige Umgebung geraten, in der Neustrukturierung von Themen gefangen sein, als Sündenbock herhalten müssen, an der Heimatfront gespalten sein oder mit irgendeinem Albtraum konfrontiert werden. Letztendlich muss sie möglicherweise für viele Jahre mit einem in hohem Maße unerwünschten EU-Ergebnis leben. Daher verhalten sich professionelle Gruppen, ähnlich wie Soldaten, die in einem riskanten Feld überleben wollen, klug und umsichtig, indem sie sich bewusst vorbereiten und vorsichtig handeln. Der zweite Grund für ihr umsichtiges Verhalten besteht darin, dass sich eine kluge Lobbygruppe ihre Lizenz zur Ausübung ihrer Tätigkeit in zukünftigen und als relevant betrachteten Arenen sichern möchte. Die umsichtige Lobbygruppe mag zuweilen Teile ihres zur Debatte stehenden Interesses verlieren, aber sie kann dennoch den Respekt, den ihr andere entgegenbringen, sowie ihren Rückhalt an der Heimatfront bewahren. Würde sie durch ihre Nachlässigkeit Gegenstand einer Kontroverse oder eines Skandals werden, würde sie zur selben Zeit oder in naher Zukunft auch aus jeder anderen Arena herausfliegen und mit an Sicherheit grenzender Wahrscheinlichkeit mehrere Interessen verlieren.

\section{Zwei Schlussfolgerungen}

Eine allgemeine Schlussfolgerung aus all diesem ist, dass professionelle Lobbygruppen höchst erfolgreich in der Beeinflussung der EU sind und wohl oder übel in hohem Maße zur EUDemokratie beitragen, da sie andere respektieren, sich umsichtig verhalten und die systemgebundenen Korrekturmechanismen stärken. Somit ist „mehr Machiavelli“ besser als weniger. Nonchalante Gruppen machen zu ihrem eigenen Nachteil mehr Fehler und tragen, indem sie gegnerische Interessen und die gesellschaftliche Kontrolle missachten, entweder überhaupt nicht oder aber negativ zur EU-Demokratie bei. Sie können von den Profis oder durch Versuch und Irrtum lernen, um rechtzeitig sowohl zu ihrem eigenen Erfolg als auch ein wenig zur Demokratie beizutragen. Die vielen Interessengruppen, die, aus welchem Grund auch immer, passiv bleiben und sich über EU-Ergebnisse beklagen, haben den denkbar negativsten Einfluss auf die EU-Demokratie, da sie mehr Raum für die geringere Anzahl aktiver Gruppen lassen, die an den Tischen sitzen und sie auf ihre Speisekarte setzen könnten. In einer partizipativen Demokratie sollten sie nicht die EU, sondern sich selbst beschuldigen.

Die zweite allgemeine Schlussfolgerung ist, dass die Blüte des Studiums des PAM, die in hohem Maße von einer offenen und wettbewerbsfähigen Demokratie abhängig ist, ebenfalls erheblich zu dieser beiträgt. Die Resultate dieses Studiums helfen vielen weiteren Gruppen, ihr Lobbying in der EU in einer aktiven, etablierten und professionellen Art und Weise zu betreiben und die Möglichkeit zu verringern, dass nur einige wenige Gruppen Spiele, Sätze und Partien gewinnen. Schlussendlich hängt die partizipative Demokratie der EU von vielen Gruppen und letzten Endes von vielen Personen ab, die besser wissen, wie man partizipiert und professionell Lobbying betreibt. Das wichtigste Werkzeug für den Erwerb dieser Expertise ist die organisierte mentale Kapazität. Dank der Natur haben die meisten Menschen mehr oder weniger dasselbe Gehirnvolumen, das das Rohmaterial für diese Kapazität darstellt. Es steht ihnen natürlich frei, diese Kapazität nicht ausreichend oder gar nicht zu nutzen und schlecht organisiert zu bleiben. Solche Personen sollten sich nicht über unerwünschte EU-Ergebnisse beklagen, sondern sich um ihre eigene Ignoranz und Nonchalance kümmern.

\section{Literatur}

Dagger, S. \& Kambeck, M. (Hrsg.) (2007). Politikberatung und Lobbying in Brüssel. Wiesbaden: VS.

Harris, P. \& Fleisher, C. (Hrsg.) (2005). Handbook of Public Affairs. London: Sage.

PARG (1981). Public Affairs Offices and Their Functions. Boston University.

Siegele, J. (2007). Lobbying. Wien: Facultas, WUV.

Van Schendelen, R. (2008). Brussels as the Place for Lobbying the EU. In R. de Groof (Hg.), Brussels and Europe (S. 197-214). Brüssel: ASP.

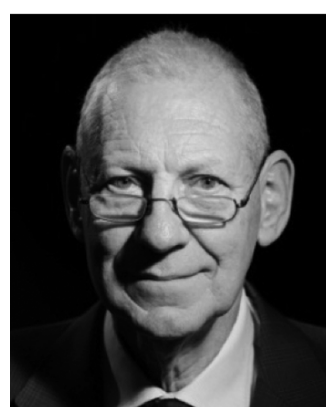

Rinus van Schendelen ist Professor für Politikwissenschaft an der Erasmus Universität in Rotterdam, Niederlande. Darüber hinaus ist er als Berater für Unternehmen und Wirtschaftsverbände im Bereich Public Affairs in der EU tätig und Mitglied verschiedener Forschungsnetzwerke. 